\title{
A New Scientific Definition of Sickness and Healing
}

\author{
Zhi Gang Sha \\ Institute of Soul Healing and Enlightenment, Richmond Hill, Canada \\ Rulin Xiu \\ Hawaii Theoretical Physics Research Center, Hawaii, USA \\ Institute of Soul Healing and Enlightenment, Richmond Hill, Canada
}

\begin{abstract}
Modern medicine has made great progress in treating diseases and prolonging life. However, modern medicine often only treats the symptoms of a disease and does not address the root cause. Based on our previous work on a mathematical definition of life, we propose a new scientific definition for sickness as the drastic increase of negative information and decrease of positive information in a certain aspect, a certain part, and a certain area of a living system. The healing process increases positive information and decreases negative information. We show that this new insight about sickness and healing can help to heal more sicknesses at the root cause, and can facilitate the development of a new science of healing.
\end{abstract}

Keywords: definition of sickness, definition of healing, positive information, definition of life, purpose of life, negative information, science of healing, science of life

\section{Introduction}

Modern medicine has made many breakthroughs that have significantly prolonged life. However, modern medicine mostly addresses and controls symptoms, and does not cure a sickness at the root cause. Furthermore, modern natural science has not been able to provide a proper conceptual framework to study the effect of consciousness on health and well-being.

In our previous work (Sha \& Xiu, 2018-1), we show that, from the quantum physics point of view, everyone and everything is the vibrational field which carries three elements: matter, energy, and information. Here, we regard matter as what we observe in the physical reality, such as our body and its cells, organs, molecules, and atoms. Energy is what moves and changes matter. Information gives shape and form to matter and energy. Information has three aspects: content of information, receiver of information, and processor of information. We suggest the content of information relates to our soul and spirit, the receiver of information relates to the spiritual heart, and the processor of information relates to our mind. We discovered that this insight can explain spiritual phenomena and wisdom in a scientific way within the framework of quantum physics. For instance, we find that these concepts can explain why and how our physical reality is manifested by our soul, spiritual heart, and mind. It explains why and how clairvoyance, telepathy, and spiritual healing work and how to develop these abilities.

Zhi Gang Sha, Dr., Institute of Soul Healing and Enlightenment, Richmond Hill, Canada.

Rulin Xiu, Ph.D., Research Director, Hawaii Theoretical Physics Research Center, Hawaii, USA; Institute of Soul Healing and Enlightenment, Richmond Hill, Canada. 
To study and describe life in a quantitative way, in our recent work (Sha \& Xiu, 2018-2) we introduce the concept of positive information and negative information into physics. We define positive information as the order a system has within itself and the degree of connection that a system has with other systems. In contrast to positive information, negative information is the disorder and degree of disconnection of a system. Negative information corresponds to the concept of entropy already defined and studied in current science. In Sha and Xiu (2018-2), we present a method to calculate positive information and the mathematical formula for positive information. In Sha and Xiu (2018-3), we explore how to use positive information to study a living system. We suggest that positive information provides a way to give life a quantitative definition. We propose that life is a system that can maintain, enhance, and develop positive information. This leads to the natural conclusion that the purpose of life is to enhance and develop more positive information. We suggest that positive information may play a significant role in studying living systems and phenomena.

In this paper, we show that the concept and understanding of positive information and negative information can give us a deeper understanding and definition of sickness. We propose that we can scientifically define sickness as the state having a drastic increase of negative information and decrease of positive information. This insight explains how consciousness and environment affect our health and how some types of spiritual healing work. It also expands the concept of sickness beyond physical sickness to emotional, mental, and spiritual sickness as well as sickness in relationships, finances, career, and more. It can also help us discover more advanced healing methods that can remove all sickness at the root cause.

\section{Scientific Definition of Sickness and Healing}

In our previous work, we define life as a system that can maintain, develop, and enhance positive information. Positive information is the order and connection within a system. It is easy to see that order and connection is what gives rise to good health, good relationships, and success in business, career, and every aspect of our lives. Negative information, or disorder and disconnection, manifest as sickness, difficulties, and challenges in our lives. We can thus define sickness in the following scientific way: "Sickness in a living system is the drastic increase of negative information and decrease of positive information within a certain aspect, a certain area, or a certain part of the body of the living system".

This scientific definition for sickness is significant in three ways. First, this definition for sickness includes not only physical sickness, but also emotional, mental, and spiritual sickness, as well as difficulties and challenges in relationships, finances, career, and more. Second, this definition of sickness tells us directly the root cause of all sickness, difficulties, and challenges. They are due to the lack of positive information and increase of negative information. Third, this new definition of sickness can explain why and how our consciousness can manifest sickness. For instance, suppose one thinks one is loved by everyone and everything. This state of consciousness will lead one to connect at deeper levels with everyone and everything, and an increase in one's positive information. This in turn will make one healthier and have better relationships, finances, and more success in one's life. On the other hand, if one thinks and feels that one is not loved by others and the world, this will reduce one's connection with others and the world. This will increase one's negative information and lead to sickness in health and difficulties in relationships, finances, career, and other aspects of life.

Based on the above scientific definition of sickness, we can easily give the following scientific definition for healing: "To heal is to change the negative information in a system into positive information". 
From this insight about sickness and healing, we can understand and explain why and how different kinds of healing modalities work. For instance, current western medicine treats the physical body to enhance positive information. Traditional Chinese medicine mostly balances energy to increase positive information.

One can also heal all sickness including improvements in health, relationships, finances, and intelligence by enhancing positive information and reducing negative information through directly changing one's soul, spiritual heart, and mind. Let us call them soul healing, heart healing, and mind healing, respectively.

Soul is the content of information. Everyone and everything is the vibrational field according to quantum physics. Our vibrational field contains a lot of information not in our conscious awareness, including information about what we have done and what our parents and ancestors have done. This hidden information secretly controls our life. To change our life, we need to transform the hidden negative information within us into positive information. We can thus define soul healing as: "Soul healing is the enhancement of positive information by changing the content of information in one's vibrational field".

The placebo effect is one of the most basic forms of soul healing. Tests of the efficacy of a new medical treatment compare the use of a given treatment to a fake treatment. The fake treatment is called a "placebo". Many research studies have found the placebo to be a valuable treatment for many sicknesses. The placebo effect is the most basic kind of soul healing. In the placebo treatment, patients receive the positive message that they have received medical treatment. The efficacy of the placebo for healing demonstrates that giving positive information for soul healing is a valuable treatment.

More advanced soul healing techniques include chanting, evocation, praying, downloads, transmissions, forgiveness practice, and distant healing.

Spiritual heart is the receiver of information from the soul. We can thus define heart healing as the enhancement of positive information by receiving positive information. Feeling and receiving love is a good way to heal all sickness. Offering and receiving forgiveness, compassion, light, humility, harmony, gratitude, and service are also powerful ways of heart healing.

Mind is the processor of information. Mind healing enhances positive information by effectively processing information. Understanding and applying wisdom is proven an effective way to heal all sickness, including health, relationships, finances, intelligence, and more.

Soul, heart, and mind healing is different from current medical treatment in the three ways: First, soul, heart, and mind healing each address the root cause of all sickness and challenges, basically transforming negative information into positive information. Physical and energetic healing methods, such as current medicine and normal traditional Chinese medicine can only address the symptoms of sickness at the physical and energetic levels.

Second, current medical treatments usually require direct physical touch, input, or interaction. Soul, heart, and mind healing can take place over distance without direct physical intervention. Third, soul, heart, and mind healing can be applied to every aspect of one's life, including the physical, emotional, mental, and spiritual aspects of our existence, as well as our relationships, finances, and more. Current medical treatments are mostly on the physical level. Their effect on other aspects of our lives is rather limited.

\section{Developing Advanced Healing Techniques}

Our scientific definitions of sickness and healing offer not only deeper insight and a broader view about sickness and healing, but can also bring us better and more advanced healing modalities. 
For example, an advanced healing technique, Tao Calligraphy, is a special and unique Chinese calligraphy written with one stroke. Additionally, the vibrational field carrying high level positive information is downloaded into each Tao Calligraphy. When people connect with it or even better trace it, the calligraphy helps transform their negative information into positive information. Through this, Tao Calligraphy has helped people heal their own or other's physical, emotional, mental, and spiritual sickness as well as difficulties and challenges in relationships, finances, career, and every aspect of life in profound ways.

Here, we share two cases. The first case regards physical sickness. Gayle is a publisher and film-maker. One day, her daughter phoned her and told her the heartbreaking news that she was diagnosed with stage four cancers. Luckily, Gayle knew about Tao Calligraphy. She immediately got a Tao Calligraphy with special blessings for her daughter. With the help of Tao Calligraphy, her daughter became cancer free within two weeks.

David Meltzer, the president of Sports 1 Marketing Company, traced a Tao Calligraphy for 15 minutes each day before going to work with the intention of expanding his business. His business experienced exponential growth, from five employees to 55 employees within a short time.

\section{Conclusion}

In this paper, we show that sickness can be given a scientific definition as the drastic increase of negative information and decrease of positive information in a certain aspect, a certain part, or a certain area of a living system. This new definition of sickness can describe and explain not only physical sickness, emotional sickness, mental sickness, and spiritual sickness, but also the sickness in relationships, finances, career, intelligence, and every aspect of life. It also gives us deeper insight about the root cause of all sickness and difficulties in our lives. It also brings a broader and deeper insight and definition of healing as increasing positive information and decreasing negative information. This new scientific definition and insight about healing can explain why the placebo effect and spiritual healing practices, such as chanting, reciting positive messages, and praying work. It also helps us develop better healing approaches that can heal all sickness at the root cause. We show case studies of one such healing technique, Tao Calligraphy. We will present further studies and research on this in our future work.

\section{References}

Sha, Z. G., \& Xiu, R. L. (2018-1). Composition of existence and the definition of spirit, heart, and mind according to quantum physics. International Journal of Information Research and Review, 5(1), 5072-5075.

Sha, Z. G., \& Xiu, R. L. (2018-2). Definition and calculation of positive information. International Journal of Information Research and Review, accepted to be published.

Sha, Z. G., \& Xiu, R. L. (2018-3). Positive information and definition of life. International Journal of Current Research in Life Sciences, accepted to be published. 